\title{
EVALUACIÓN DE TÉCNICAS DE IMPLANTACIÓN EN ZAPALLITO REDONDO DE TRONCO [CUCURBITA MAXIMA VAR. ZAPALLITO (CARR.) MILLÁN]
}

\author{
De Grazia, J. ${ }^{1}$; Tittonell, P. A. ${ }^{1}$; WissocQ, A. A. ${ }^{2}$; \\ FILIPPINI, O. S. ${ }^{3} \&$ ChIESA, A..$^{1,2}$
}

\begin{abstract}
RESUMEN
El zapallito redondo de tronco [Cucurbita maxima var. zapallito (Carr.) Millán] se cultiva en los cinturones verdes y zonas de primicias del noroeste y noreste de Argentina. Para comparar las técnicas de implantación de cultivo en siembra directa y transplante, y evaluar su efecto sobre la precocidad y el rendimiento de diferentes materiales genéticos, se realizó un ensayo considerando como tratamientos las dos técnicas de implantación mencionadas anteriormente combinadas con los cultivares Any y Any Plus, y el híbrido Ángelo $\mathrm{F}_{1}$. El diseño experimental fue bloques completos aleatorizados con cuatro repeticiones por tratamiento. Los resultados mostraron la mayor precocidad para el híbrido Ángelo $\mathrm{F}_{1}$ con transplante. Esa misma combinación de tratamientos presentó la mayor cantidad de frutos por planta. En cambio, con el cultivar Any Plus mediante transplante se observaron los frutos de mayor peso a la cosecha (madurez comercial), pero sin diferenciarse estadísticamente del resto debido a la existencia de interacción significativa genotipo $\mathrm{x}$ técnica de implantación.

Palabras claves: siembra directa, transplante, precocidad, rendimiento.
\end{abstract}

\section{SUMMARY}

\section{Evaluation of crop establishment techniques in summer squash [Cucurbita maxima var. zapallito (Carr.) Millán].}

Summer squash [Cucurbita maxima var. zapallito (Carr.) Millán] is grown in the green 'belts' around the cities and NW and NE earliness areas of Argentina. The effect of the direct sowing and transplanting on earliness and yield with different genotypes was evaluated. Treatments were the two crop establishment techniques combined with the cultivars Any and Any Plus, and the hybrid Angelo $\mathrm{F}_{1}$. A randomized block-design was used with four replicates. Hybrid Angelo $F_{1}$ by transplanting showed earliness and the higher fruits number per plant. On the other hand, Any Plus by transplanting showed the highest weight of fruits at harvest (commercial maturity) without statistical differences because of a significant interaction genotype $\mathrm{x}$ crop establishment technique.

Key words: direct sowing, transplanting, earliness, yield.

1.- Cátedra de Horticultura y Floricultura, Facultad de Ciencias Agrarias, Universidad Nacional de Lomas de Zamora. Ruta Provincial No 4, km 2. (1836) Llavallol, Buenos Aires. E-mail: jadegazia@yahoo.com degrazia@agrarias.net

2.- Cátedra de Horticultura, Facultad de Agronomía, Universidad de Buenos Aires. Av. San Martín 4453,

(1417) Ciudad Autónoma de Buenos Aires.

3.- Cátedra de Estadística, Dpto. de Ciencias Básicas, Universidad Nacional de Luján. Cruce de Rutas Nacionales $\mathrm{N}^{\circ} 5$ y N $\mathrm{N}^{\circ}$ 7. (6700) Luján, Buenos Aires. C. C. 221.

Manuscrito recibido el 17 de diciembre de 2004 y aceptado para su publicación el 10 de febrero de 2005. 